\title{
Global stability of Vegas-like TCP flow
}

\author{
Hyojeong Choe \\ EE, Caltech/Postech \\ hjchoe@caltech.edu
}

\author{
Steven H. Low \\ $\mathrm{CS}$ and EE, Caltech \\ slow@caltech.edu
}

Jin S. Lee

EE, Postech

jsoo@postech.ac.kr

\begin{abstract}
A TCP Vegas flow adapts its sending rate to maintain a constant backlog in its path. The stability of nonlinear adaptation has been analyzed based on linearization and only accounted for a small signal. In this paper, we extend the error model of TCP-like flow to a state-dependent coefficient form with nonlinear state feedback. The nonlinear feedback is here approximated by a saturation function. Using a quadratic Lyapunov function approach, we find a domain of attraction to show that the unique equilibrium point of the system is asymptotically stable in the domain.
\end{abstract}

\section{FLOW-LEVEL MODEL FOR TCP VEGAS}

Congestion avoidance algorithm of TCP Vegas [1] is modelled as a flow-level dynamics by [6], [5], [2]. For a general network of Vegas, [6], [5] show that the equilibrium is weighted proportionally fair. Sufficient conditions for the local asymptotic stability of Vegas and its modification in the presence of heterogenous feedback delays are derived in [2]. The work accounts for local behavior around equilibrium.

On the global stability, however, there are very few studies have been done because the coupling between primal and dual variables complicates the analysis. See [7], [3], [11], [10] on nonlinear stability analysis for other congestion control algorithms. We examine the global stability of a Vegas-like protocol in this paper, under the assumption that there is no feedback delay in source dynamics nor in link dynamics.

Recall the Vegas model of a single-source and singlelink network in continuous-time scale. The window size of TCP Vegas source $w$ is adapted at the feedback of the link congestion measure $p$. $p$ is queuing delay. Then a dynamic model in continuous-time scale consists of the time-average variables of both $w$ and $p$.

$$
\begin{aligned}
\dot{w}(t) & =-\frac{1}{T(t)} \operatorname{sign}[x(t) p(t)-\alpha d]_{w(t)}^{+} \\
\dot{p}(t) & =\frac{1}{c}[x(t)-c]_{p(t)}^{+} \\
x(t) & =\frac{w(t)}{T(t)} \\
T(t) & =p(t)+d
\end{aligned}
$$

where $w(t)$ is the window size of TCP flow, $p(t)$ is queuing delay at the link, $x(t)$ is the flow rate, and $c$ and $d$ are the link capacity and the flow propagation delay respectively. And

$$
[x]_{a}^{+}=\left\{\begin{array}{cc}
1 & \text { if } a>0 \\
\max \{x, 0\} & \text { if } a=0
\end{array} .\right.
$$

We assume that the subspace such that $w(t)>0$ and $p(t)>0$ is in our interest, then the dynamic model of $x(t)$ and $p(t)$ is like this:

$$
\begin{aligned}
\dot{x}(t) & =-\frac{1}{T(t)^{2}} \operatorname{sign}(x(t) p(t)-\alpha d) \\
& -\frac{x(t)(x(t)-c)}{c T(t)} \\
\dot{p}(t) & =\frac{1}{c}(x(t)-c)
\end{aligned}
$$

In this model, the flow rate update equation has an addition term $-\frac{x(t)(x(t)-c)}{c T(t)}$, compared with that in [2] where the time derivative of $q(t)$ is assumed negligible.

Given the link capacity $c$ and the propagation delay $d$, the equilibrium point of TCP Vegas is $\left(x^{*}, p^{*}\right)=(c, \mu d)$ [6], [5], where $\mu=\frac{\alpha}{c}$ is the ratio of the margin of server sending rate allowed in the link queue $\alpha$ to the available bandwidth $c$. It is easy to show that the equilibrium point is unique in the the domain of our interest. Without loss of generosity, we set an equivalent error model of TCP Vegas to move the equilibrium point to zero. And we approximate $\operatorname{sign}(u)$ to saturation function sat $\left(\frac{u}{\epsilon}\right)$, where the approximation is reasonable for a sufficiently small $\epsilon>0$ as Figure 1 shows. 


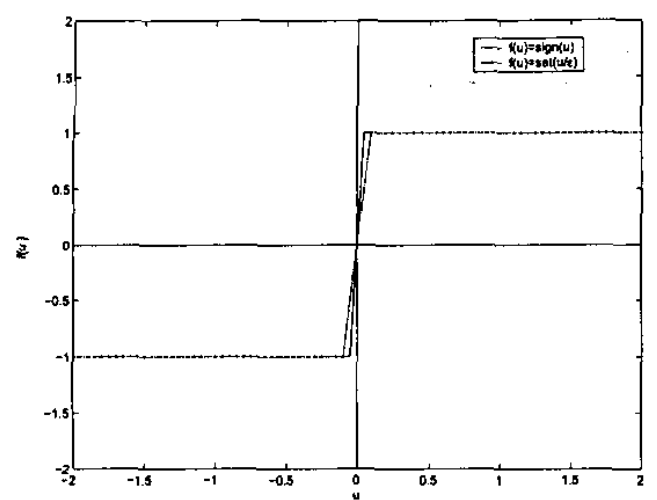

Fig. 1. Sign function is approximated to saturation $f(u)=\operatorname{sat}\left(\frac{u}{\epsilon}\right)$.

Then the error model can be set in state-dependent coefficient form with a saturated nonlinear input. Let

$$
z=\left[\begin{array}{l}
z_{1} \\
z_{2}
\end{array}\right]=\left[\begin{array}{l}
x-x^{*} \\
p-p^{*}
\end{array}\right]
$$

Given a finite queue size $Q<\infty$, the delay-based algorithm is meaningful only in the feasible domain which is free from any packet loss event. To avoid packet loss, the backlog of the Vegas flow should not exceed $Q$, i.e.

$$
\left(z_{1}+c\right)\left(z_{2}+\mu d\right) \leq Q
$$

Thus the feasible domain $D(z)$ is finally defined as

$$
\begin{aligned}
D & :=\left\{z \in \mathbb{R}^{2} \mid z_{1} \in\left(-c, x_{\max }-c\right],\right. \\
& \left.z_{2} \in\left[-\mu d, \frac{Q}{x_{\max }-c}-\mu d\right]\right\}
\end{aligned}
$$

where it is assumed that $x(t)$ has an upper-bound $x_{\max }$. Figure 2 illustrates the domain $D$.

The network model of a TCP Vegas-like flow and a Drop-Tail bottleneck is driven to a state-dependent coefficient form with nonlinear saturation input as follows.

$$
\dot{z}=A(z) z+B(z) \operatorname{sat}(F(z) z)
$$

where

$$
\begin{aligned}
& A(z)=\left[\begin{array}{cc}
-\frac{\left(z_{1}+c\right)}{(1+\mu) c d} & 0 \\
\frac{1}{c} & 0
\end{array}\right], \\
& B(z)=\left[\begin{array}{c}
-\frac{\omega}{\left(z_{2}+(1+\mu) d\right)^{2}} \\
0
\end{array}\right], \\
& F(z)=\left[\begin{array}{cc}
\frac{z_{2}+2 \mu d}{2 \epsilon} & \frac{z_{1}+2 c}{2 \epsilon}
\end{array}\right] .
\end{aligned}
$$

Here $\omega>0$ is a parameter for step size in flow rate update. This system under saturation feedback is the same

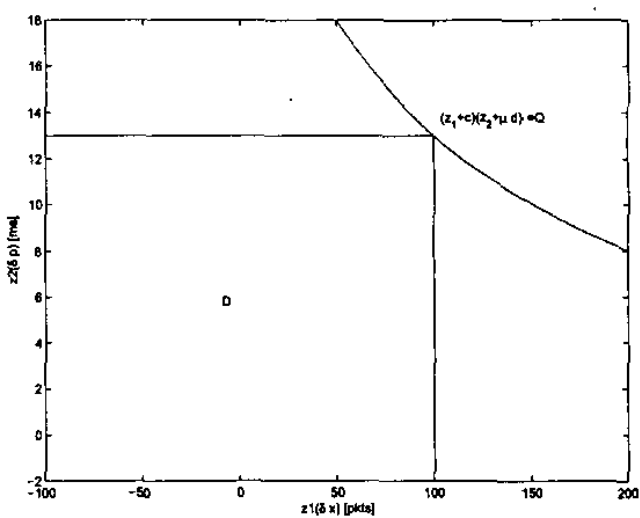

Fig. 2. The feasible domain $D(z)$ with $(c, d)=(100 \mathrm{pkts} / \mathrm{ms}, 100 \mathrm{~ms})$, $Q=3000$ pkts and $x_{\max }=200$ pkts $/ \mathrm{ms}$.

framework of [4], but the difference is that system matrices $A(z), B(z)$, and $F(z)$ are state-dependent.

\section{STABILITY ANALYSIS}

To analyze the stability of the error model (9), we set a quadratic form of Lyapunov function $V(z)=z^{T} P z$ which satisfies $V(0)=0$ and $V(z) \geq 0, \forall z \in \mathbb{R}^{2}$. For simplicity, a diagonal matrix

$$
P=\left[\begin{array}{cc}
p_{1} & 0 \\
0 & p_{2}
\end{array}\right]
$$

is chosen as Riccati matrix, where $p_{1}, p_{2}>0$ thus $P \succ$ 0 . Then $R(P, \rho)=\left\{z \in \mathbb{R}^{2} \mid V(z) \leq \rho\right\}$ is a compact set containing the origin $z=0$, for all $\rho>0$ and it is clear that the origin is the unique equilibrium in $R(P, \rho)$. Thus, we show the following theorem to extend [4] to the model (9) in the state-dependent coefficient form.

Theorem 1: Given $R(P, \rho>0)$, suppose that

$$
\begin{array}{r}
(A(z)+B(z) F(z))^{T} P+P(A(z)+B(z) F(z)) \leq 0, \\
\forall z \in R(P, \rho) .
\end{array}
$$

Then, $R(P(z), \rho>0)$ is invariant under the closedloop system (9), if there exists a row matrix $H(z)=$ $\left[h_{1}(z) h_{2}(z)\right] \in \mathbb{R}^{1 \times 2}$ such that

$$
\begin{array}{r}
(A(z)+B(z) H(z))^{T} P+P(A(z)+B(z) H(z))<0, \\
\forall z \in R(P, \rho>0)
\end{array}
$$

and

$$
R(P, \rho>0) \subset \mathcal{L}(H(z))
$$

where $\mathcal{L}(H(z))$ is the linear region of sat $(H(z) z)$, i.e. $\mathcal{L}(H(z)):=\left\{z \in \mathbb{R}^{2}|| H(z) z \mid \leq 1\right\}$. Furthermore, if $z=$ 
0 is one and only point that stays forever among $\forall z \in S_{F}$ defined as

$$
\begin{aligned}
S_{F} & :=\left\{z \in R(P, \rho>0) \mid(A(z)+B(z) F(z))^{T} P\right. \\
& +P(A(z)+B(z) F(z))=0\}
\end{aligned}
$$

and $\forall z \in S_{H}$ defined as

$$
\begin{aligned}
S_{H} & :=\left\{z \in R(P, \rho>0) \mid(A(z)+B(z) H(z))^{T} P\right. \\
& +P(A(z)+B(z) H(z))=0\}
\end{aligned}
$$

, any trajectory $z(t)$ of (9) with $z(0) \in R(P, \rho>0)$ remains in $R(P, \rho>0)$ for all $t \geq 0$ and converges to the origin as $t$ goes to $\infty$.

Proof: Before determining the invariance of $R(P(z), \rho)$, we first make sure that (14) is satisfied under the system (9).

$$
\begin{aligned}
(A(z)+B(z) F(z))^{T} P & +P(A(z)+B(z) F(z)) \\
& \leq 0, \forall z \in R(P, \rho>0) .
\end{aligned}
$$

we, at first, examine the inequality at $z=0$. Let

$$
\begin{aligned}
& A_{0}=A(0) \\
& B_{0}=B(0) \\
& F_{0}=F(0) .
\end{aligned}
$$

Then, with $P=I$,

$$
\begin{aligned}
\left(A_{0}\right. & \left.+B_{0} F_{0}\right)^{T} P+P\left(A_{0}+B_{0} F_{0}\right) \\
& =\left(\begin{array}{cc}
-\frac{\epsilon(1+\mu) c+\omega \mu}{\epsilon(1+\mu)^{2} d^{2}} & -\frac{\omega c^{2}-\epsilon(1+\mu)^{2} d^{2}}{\epsilon(1+\mu)^{2} c d^{2}} \\
-\frac{\omega c^{2}-\epsilon(1+\mu)^{2} d^{2}}{\epsilon(1+\mu)^{2} c d^{2}} & 0
\end{array}\right) \\
& \prec 0 .
\end{aligned}
$$

For $z \neq 0$, there exists $\rho>0$ such that the numerator on the left hand side of (17) turns out to be a sum of squares of polynomials in $\left(z_{1}^{2}, z_{1} z_{2}, z_{2}^{2}, z_{1}, z_{2}, 1\right)$ ([8], [9]) by choosing a reasonable parameter set. On the other hand, we always have $R(P(z), \rho) \subset \mathcal{L}(\tilde{H}(z))$, i.e. $|H(z) z| \leq 1$, $\forall z \in R(P(z), \rho)$ therefore there exists an $\vec{H}(z) \in \mathbb{R}^{2}$ which satisfies (15) and (16). Thus the sufficiency holds. It is clear that among all $z \in S_{F} \cup S_{H}$, only $z=0$ satisfies $\dot{z}(t)=0$. Combining La Salle's theorem, we can conclude that these (14)-(16) are the sufficient conditions for the nonlinear asymptotic stability of (9).

\section{THE REgION OF ATTRACTION}

Given a network topology $(c, d)$, we can abstract the explicit conditions of design parameters $\mu=\frac{\alpha}{c}, \epsilon$, and $\omega$, and the positive matrix coefficients $p_{1}$ and $p_{2}$ for the Vegaslike protocol to maximize the estimated region of attraction defined by $\rho$, so that it covers all $z$ in the feasible domain $D$ in Figure 2.

From Theorem 1, the following inequality condition can be derived:

$$
\begin{aligned}
\left(2 \mu c d p_{1}\right) z_{2}^{4} & +\left\{c\left(z_{1}+x^{*}\right) p_{1}-\frac{\left(d+\mu d+z_{2}\right)^{2} p_{2}}{\omega}\right\} z_{1} z_{2} \\
& +\mu c d p_{2} z_{2}^{2} \\
& >0
\end{aligned}
$$

Note that (19) is not always satisfied in the global space $\mathbb{R}^{2 \times 2}$. However, it holds for a feasible set $D$ in $\mathcal{R}$, if we choose $\omega$ and $\epsilon$ and the quadratic Lyapunov function parameters $p_{1}$ and $p_{2}$ such that

$$
\frac{2 \epsilon}{\omega} \sqrt{\frac{p_{2}}{2 p_{1}}}<\frac{c}{\bar{d}}
$$

and choose $\mu$ such that

$$
\mu \geq s-\sqrt{s^{2}-1},
$$

where $s:=\frac{\omega}{\epsilon} \sqrt{\frac{2 p_{1}}{p_{2}}} \frac{c}{d}-1>1$ is lower-bounded by (20).

\section{CONCLUSION}

We have modelled a network with Vegas-like TCP as a standard-dependent coefficient form with saturated nonlinear state feedback, and examined the nonlinear stability of a single-link single-source model in the framework of invariant system. The analysis derives a sufficient condition for expanding the region of attraction to the feasible domain of our interest. For more general network topologies, the nonlinear global stability of TCP Vegas or the class of similar delay-based protocol is still an interesting open problem. The effect of boundary constraints and delay is also worth going into further research.

\section{REFERENCES}

[1] Lawrence S. Brakmo and Larry L. Peterson. TCP Vegas: end-to-end congestion avoidance on a global Internet. IEEE Joumal on Selected Areas in Communications, 13(8):1465-80, October 1995. http: //cs.princeton. edu/nsg/papers/j sac-vegas.ps.

[2] H. Choe and S. H. Low. Stabilized Vegas. In Proc. of IEEE Infocom. http://netlab. caltech.edu.

[3] S. Deb and R. Srikant. Global stability of congestion controllers for the Internet. IEEE Transactions on Automatic Control, 48(6):10551060, June 2003. 
[4] Tingshu $\mathrm{Hu}$ and Zongli Lin. Exact Characterization of Invariant Ellopsoids for Single Input Linear Systems Subject to Actuator Saturation. IEEE Transactions on Automatic Control, Vol. 47, No.1.

[5] Steven H. Low, Larry Peterson, and Limin Wang. Understanding Vegas: a duality model. J. of $A C M, 49(2): 207-235$, March 2002. http: //netlab.caltech. edu.

[6] Jeonghoon Mo and Jean Walrand. Fair end-to-end windowbased congestion control. IEEE/ACM Transactions on Networking, $8(5): 556-567$, October 2000

[7] Fernando Paganini. A primal-dual congestion control with stability, high utilization and arbitrary source utilities. Preprint, April 2002.

[8] P. Parrilo. Structured semidefinite programs and semialgebraic geometry methods in robustness and optimization. California Institute of Technology, 2000. PhD thesis.

[9] P. Parrilo. Semidefinite programming relaxations for semialgebraic problems. 2002. submitted to Mathematical Programming.

[10] T. Basar T. Alpcan. A utility-based congestion control scheme for internet-style networks with delay. In Proc. of Infocom 2003.

[11] Z. Wang and F. Paganini. Global stability with time-delay in network congestion. In Proc. of the CDC. 